\title{
BIORREMEDIAÇÃO DE SOLO CONTAMINADO POR BIODIESEL BRUTO EM REATOR ROTATIVO: AMPLIAÇÃO NA ESCALA DE TRATAMENTO
}

\author{
RAMINHO, M. L. F. ${ }^{1}$, V. L. CARDOSO ${ }^{1}$, U.C. FILHO $^{1}$, CRUZ, M. L. ${ }^{1}$, T. G. BRAGA ${ }^{1}$ \\ ${ }^{1}$ Universidade Federal de Uberlândia, Faculdade de Engenharia Química \\ E-mail para contato: marcelafanni@yahoo.com.br
}

\begin{abstract}
RESUMO - A preocupação com possíveis contaminações provocadas por acidentes durante o processamento do biodiesel tem se refletido no crescente interesse da comunidade científica em estudos sobre tratamentos viáveis para o ambiente contaminado por este produto. Pesquisas com biorremediação de solo contaminado têm sido encontradas na literatura mostrando resultados sólidos que permitem afirmar que a tecnologia é promissora, porém, é necessário desenvolver o processo em escala piloto para avaliar a viabilidade técnica do mesmo. Neste trabalho foi realizada a biorremediação de um solo contaminado com 77.400 PPM de biodiesel bruto, em escala piloto com capacidade de processamento de $22 \mathrm{~kg}$, utilizando reator rotativo com controle de umidade e adição de nutrientes. Após 42 dias de processo, os resultados de degradação do biodiesel obtidos neste trabalho foram semelhantes aos resultados encontrados para o mesmo tipo de tratamento em escala laboratorial.
\end{abstract}

\section{INTRODUÇÃO}

O desenvolvimento de métodos destinados ao tratamento de ambientes (solo e água) contaminados é uma atividade importante que acompanha a criação de novos processos industriais e a utilização de novas fontes de energia. A biorremediação é uma alternativa promissora para o tratamento de solos contaminados com óleos combustíveis, pois se mostra como uma forma sustentável e ambientalmente correta (El Fantroussi e Agathos, 2005), além de possuir várias aplicações, baixos custos operacionais e produzir bons resultados (Bento et al., 2005). A biorremediação é um processo de tratamento que utiliza microrganismos para transformar substâncias perigosas presentes no ambiente em substâncias menos ou não tóxicas, através de processos de degradação biológica (Usepa, 2004). Porém, a efetividade da biorremediação em determinada situação não garante que a mesma seja efetiva em outra situação, pois esta depende da interação de diversos fatores como a dinâmica populacional microbiana do solo, características nutricionais do solo, variações de $\mathrm{pH}$, umidade, variações de temperatura e composição do meio poluente.

Entre os novos combustíveis de interesse em grande escala no Brasil e em diversos países está o biodiesel que é um combustível obtido a partir de matérias-primas vegetais ou animais que vem se destacando como alternativa para substituição parcial de derivados do petróleo de forma sustentável visando reduzir a poluição ambiental. No Brasil, a produção de biodiesel possui 
importantes vantagens devido à grande disponibilidade de matéria-prima e ao crescimento contínuo da indústria de óleos vegetais e de etanol (Rivaldi et al., 2007).

Os investimentos crescentes na produção de biodiesel geram preocupações com as possíveis contaminações provocadas por derrames ou outros acidentes durante o processamento do mesmo. Devido ao exposto tem-se um grande interesse da comunidade científica em estudos sobre biorremediação para o ambiente (solo e água) contaminado por biodiesel. Embora seja crescente este interesse há na literatura escassez de trabalhos que descrevam este tratamento em equipamentos de dimensões maiores que a escala de bancada. Diante desta situação, o presente trabalho se dedica ao estudo da biorremediação de solo contaminado por biodiesel bruto, utilizando reator rotativo tipo batelada, em escala piloto.

\section{MATERIAIS E MÉTODOS}

\subsection{O reator e a biorremediação do solo}

O equipamento utilizado no experimento foi um reator rotativo com capacidade de $45 \mathrm{~kg}$, rotação de 20 RPM e abertura para amostragem conforme mostrado na Figura 1. O reator foi utilizado com carga de $22 \mathrm{~kg}$ de solo coletado na Fazenda do Glória, pertencente à Universidade Federal de Uberlândia (Uberlândia-MG) que foi misturado a biodiesel bruto cedido pela Petrobrás Transporte S/A, sediada em Uberlândia-MG.

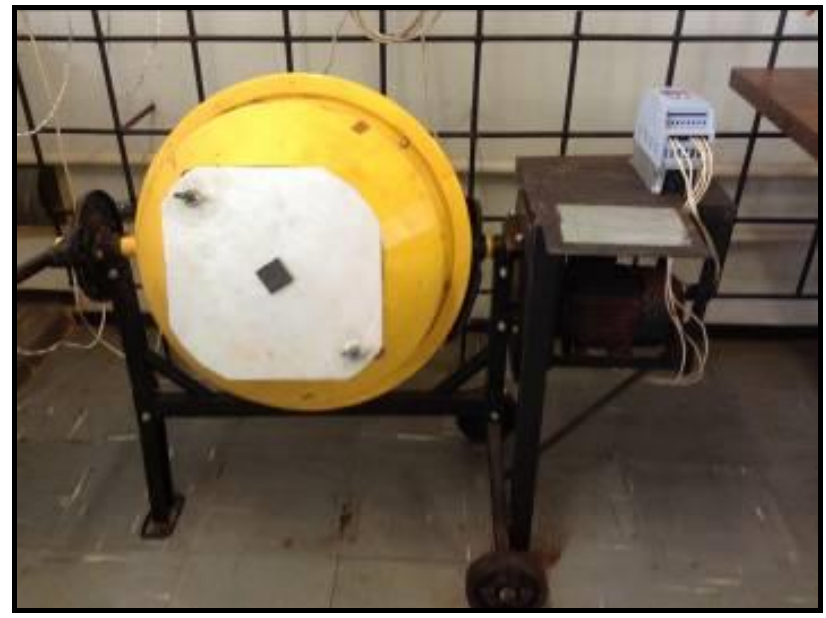

Figura 1 - Sistema reator rotativo.

Para o experimento de biorremediação, biodiesel bruto foi adicionado ao solo na concentração de $77.400 \mathrm{mg} \mathrm{kg}^{-1}$ e este mesmo solo foi inoculado com cultura mista isolada do solo de lagoa usada no tratamento de derivados de petróleo (Vieira, 2004) na proporção de $10^{8}$ células $\mathrm{mg}^{-1}$ de solo. Na operação do reator foi realizada a correção da umidade do solo para $14 \%$ a cada sete dias e a correção, no mesmo período de tempo, da relação dos nutrientes $\mathrm{C}$ (carbono), $\mathrm{N}$ (nitrogênio) e P (fósforo) na proporção de 100:10:1. Também foi realizado o monitoramento da temperatura. 


\section{9 a 22 de outubro de 2014 \\ Florianópolis/SC}

\subsection{Características do solo}

A análise da capacidade de retenção de água pelo solo foi realizada colocando a amostra em filtro de papel e umedecendo a mesma até a sua saturação. Em seguida, o conjunto foi pesado e colocado em uma estufa a $100 \pm 5^{\circ} \mathrm{C}$, por um período de vinte e quatro horas. Após este tempo, o conjunto foi resfriado em um dessecador até que a temperatura ambiente fosse alcançada. Pesou-se novamente o conjunto e determinou-se o conteúdo de umidade (capacidade de retenção de água), relacionando a perda de massa inicial com a massa final da amostra, multiplicando-se por 100 para obter-se o resultado em porcentagem (Watwood et al., 1991).

A distribuição granulométrica do solo foi determinada no Laboratório de Geotecnia da Faculdade de Engenharia Civil da Universidade Federal de Uberlândia. O ensaio foi executado, seguindo-se a ABNT (NBR 7181/1984), que compreende as etapas de homogeneização da amostra, secagem forçada, passagem por uma série de peneiras e por fim, sedimentação em solução.

\subsection{Métodos analíticos}

O percentual de biodiesel foi medido através de análise de Hidrocarbonetos Totais de Petróleo (TPH). A extração do biodiesel do solo foi feita com solvente hexano e posteriormente analisado em analisador de Hidrocarbonetos Totais de Petróleo (TPH) Modelo Buck HC-404 via curva de calibração conforme metodologia descrita em Vieira et al. (2007).

A quantificação de fósforo foi realizada pelo método colorimétrico por redução com ácido ascórbico. A análise de nitrogênio total foi realizada pelo método Kjeldahl e o carbono pela técnica de combustão catalítica a alta temperatura, empregando o aparelho Total Organic Carbon Analyzer - Module SSM5000A - Shimadzu. A correção do nitrogênio foi feita com Extrato de Levedura e $\mathrm{NH}_{4} \mathrm{NO}_{3}$ e a correção do fósforo foi feita com $\mathrm{K}_{2} \mathrm{HPO}_{4} \mathrm{e} \mathrm{KH}_{2} \mathrm{PO}_{4}$.

A determinação da quantidade de bactérias no solo, foi feita através da mistura do solo em solução salina $(0,9 \% \mathrm{NaCl})$ na proporção de $10 \mathrm{~g}$ de solo para $100 \mathrm{~mL}$ de solução. Esta mistura foi submetida a diferentes diluições e plaqueada utilizando a técnica 'pour plate' (Apha, 1992) em meio de cultura de composição (g/L): ágar $(15,0)$, peptona de carne $(10,0), \mathrm{NaCl}(5,0)$, extrato de carne $(3,0)$ e $\mathrm{K}_{2} \mathrm{HPO}_{4}(1,0)$. As placas foram incubadas a $30 \pm 5^{\circ} \mathrm{C}$ por 48 horas antes da contagem de unidades formadoras de colônias.

O cálculo da umidade do solo foi feito pela diferença da massa inicial e final da amostra, após permanência em estufa a $100 \pm 5^{\circ} \mathrm{C}$ por um período de vinte e quatro horas, multiplicandose por 100 para se obter o resultado em percentagem, conforme a Norma Brasileira - ABNT (NBR 7180/1984).

A análise de acidez do solo foi realizada homogeneizando-se $10 \mathrm{~g}$ de solo e $25 \mathrm{~mL}$ de água destilada por um período de 30 minutos com auxílio de um agitador magnético. Decorrido o 
período de agitação, a mistura foi deixada em repouso para separação das fases e procedeu-se com a leitura do $\mathrm{pH}$ do solo, através do decantado, em potenciômetro.

\section{RESULTADOS E DISCUSSÃO}

A capacidade de retenção de água medida conforme metodologia citada anteriormente foi de $28 \%$. Assim, a biorremediação foi iniciada com $14 \%$ de umidade e a cada sete dias foi feita a correção da umidade para este valor, pois a umidade ótima para biodegradação de hidrocarbonetos segundo Meyer (2011) encontra-se na faixa de 25 e $85 \%$ da capacidade de retenção de água.

Na Tabela 1 são mostrados os resultados da caracterização físico-química, além da composição granulométrica da amostra de solo utilizada. Ao analisar os dados de granulometria, pode-se classificar o solo empregado neste estudo como argila arenosa (Prevedello, 1996). O pH ácido $(5,31)$ é característico da maioria dos solos brasileiros. A análise mostra ainda que este solo é extremamente pobre em nitrogênio e fósforo, elemento limitante em processos de biodegradação, mostrando a necessidade de correções.

Tabela 1 - Características do solo utilizado no experimento

\begin{tabular}{|l|l|}
\hline \multicolumn{1}{|c|}{ Característica } & \multicolumn{1}{c|}{ Resultado } \\
\hline Areia Grossa & $0,3 \%$ \\
\hline Areia média & $5,3 \%$ \\
\hline Areia Fina & $3,8 \%$ \\
\hline Silte & $35,5 \%$ \\
\hline Argila & $45,6 \%$ \\
\hline pH & $5,31 \pm 0.10$ \\
\hline Fósforo (P) assimilável (mg/kg solo) & 3,01 \\
\hline Carbono (C) orgânico (g/kg solo) & 12,5 \\
\hline Nitrogênio (N) total (g/kg solo) & 0,12 \\
\hline Relação C:N:P & $100: 0,77: 0,019$ \\
\hline
\end{tabular}

Na Tabela 2 é apresentada a concentração de biodiesel no solo ao longo dos 42 dias de funcionamento do reator. Pode-se observar que a maior remoção ocorreu entre 7 e 21 dias de experimento, sendo que a semana entre os dias 14 e 21 apresentou o resultado mais significativa e que a redução final de biodiesel no solo contaminado foi de $28,77 \%$. Os resultados obtidos são superiores ao observado por Lemos (2013) em escala de bancada a $40^{\circ} \mathrm{C}$ que cita que para uma contaminação de $70.000 \mathrm{mg} \mathrm{kg}^{-1}$ foi observado uma remoção de $19 \%$ em 42 dias.

Tabela 2 - Resultados de remoção do contaminante

\begin{tabular}{|c|c|c|c|c|c|c|c|}
\hline Número de dias & $\mathbf{0}$ & $\mathbf{7}$ & $\mathbf{1 4}$ & $\mathbf{2 1}$ & $\mathbf{2 8}$ & $\mathbf{3 5}$ & $\mathbf{4 2}$ \\
\hline $\begin{array}{c}\text { Concentração de } \\
\text { biodiesel (\%) }\end{array}$ & 7,74 & 7,53 & 7,23 & 5,75 & 5,65 & 5,52 & 5,51 \\
\hline $\begin{array}{c}\text { Remoção de biodiesel } \\
(\%)\end{array}$ & 0,00 & 2,70 & 6,55 & 25,72 & 26,97 & 28,64 & 28,77 \\
\hline
\end{tabular}


A Tabela 3 mostra a relação entre as concentrações dos nutrientes carbono, nitrogênio e fósforo em cada amostragem que antecedeu a correção da composição do meio. Podemos perceber maior consumo de nutrientes, ou seja, menor relação C:N:P, no período de 7 a 21 dias, período este que coincide com a fase de maior atividade metabólica dos microorganismos relacionada com a maior remoção do contaminante.

Tabela 3 - Relação entre as concentrações dos nutrientes C, N e P

\begin{tabular}{|c|c|c|c|c|c|c|c|}
\hline Número de dias & $\mathbf{0}$ & $\mathbf{7}$ & $\mathbf{1 4}$ & $\mathbf{2 1}$ & $\mathbf{2 8}$ & $\mathbf{3 5}$ & $\mathbf{4 2}$ \\
\hline Relação C:N:P & $100: 10: 1$ & $100: 8,02: 0,83$ & $100: 7,59: 0,87$ & $100: 6,37: 0,62$ & $100: 8,18: 0,84$ & $100: 8,35: 0,82$ & $100: 8,64: 0,91$ \\
\hline
\end{tabular}

A Figura 2 mostra a temperatura do sistema ao longo dos 42 dias de experimento. Pode-se observar que a mesma se manteve entre 34 e $36^{\circ} \mathrm{C}$ o que sugere que a remoção pode ser melhorada, pois a temperatura influencia na degradação do contaminante, sendo que a faixa de 28 a $32^{\circ} \mathrm{C}$ é citada como ideal para os microrganismos responsáveis pela biorremediação (Moreira e Siqueira, 2006).

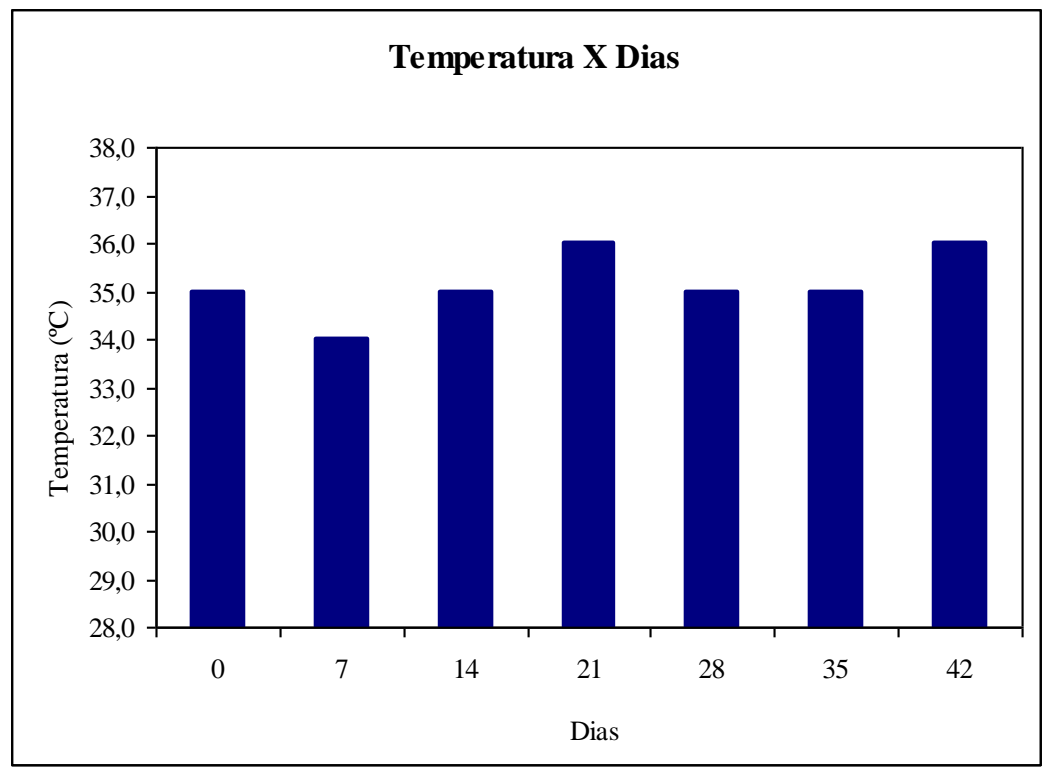

Figura 2 - Temperatura observada ao longo do experimento.

Através da Figura 3 podemos perceber que durante todo o experimento a umidade foi inferior a 14\% mesmo com a adição de água a cada sete dias para corrigir a umidade para este valor. Esta figura também mostra que a maior perda de água ocorreu no período de 7 a 21 dias, ou seja, a maior remoção de biodiesel ocorreu no período que houve maior perda de água dentro do reator. Este fato sugere que o funcionamento do reator pode melhorado com a correção da umidade em intervalos de tempos menores que sete dias. Segundo Rizzo et al. (2007) o processo de biorremediação realizado com umidade próxima a $20 \%$ produz resultados de remoção superiores ao observado neste trabalho. Jacques et al. (2010) mostrou em seu trabalho que a umidade é considerada o fator de maior importância na biorremediação do solo, visto que 
reduções relativamente pequenas da umidade resultam em significativas reduções da atividade microbiológica do solo.

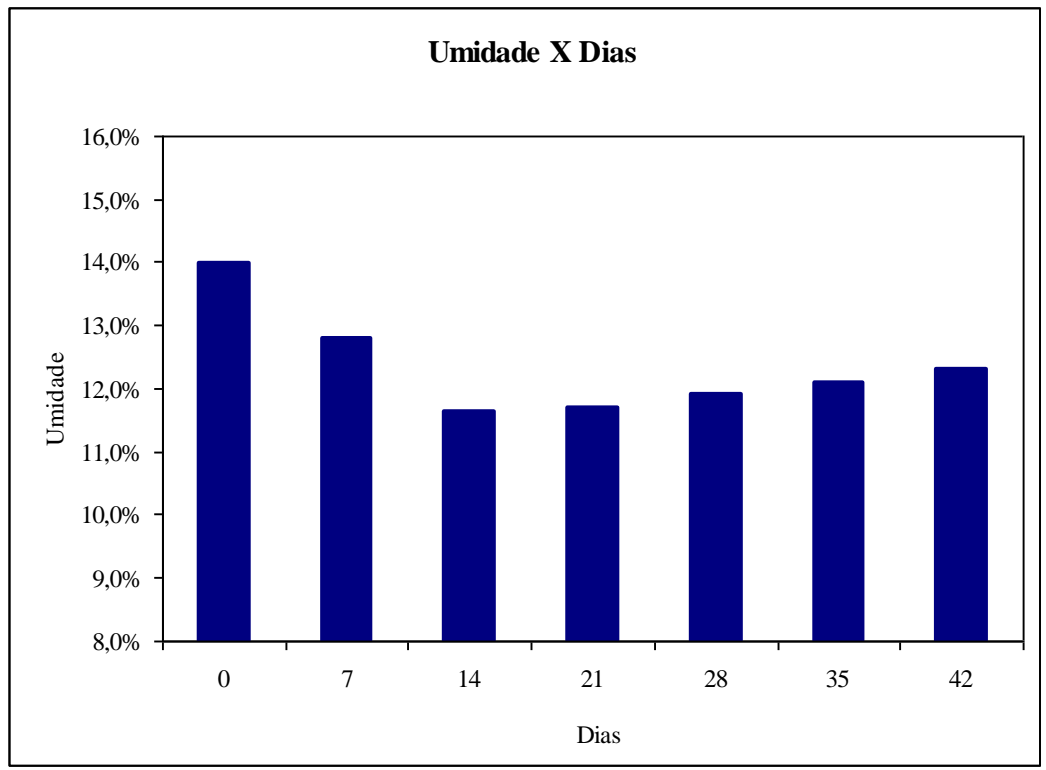

Figura 3 - Umidade medida semanalmente.

Na Figura 4 é apresentada a concentração dos microrganismos no solo. Pode-se observar houve um aumento na população microbiana do solo desde a inoculação (dia zero) chegando ao pico no $28^{\circ}$ dia o que justifica o maior percentual de remoção de biodiesel no período do $7^{\circ}$ ao $21^{\circ}$ dia de processo, conforme já discutido anteriormente.

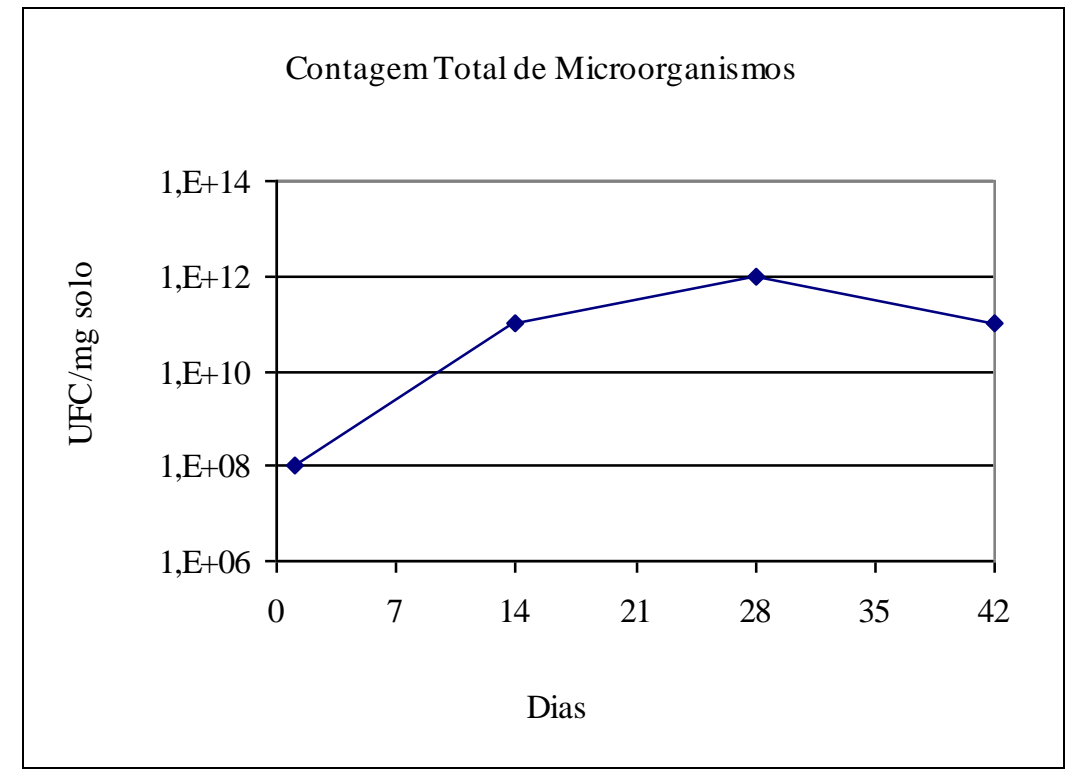

Figura 4 - Perfil da população microbiana no solo contaminado. 


\section{CONCLUSÃO}

Diante dos dados obtidos podemos concluir que: a) a utilização do biorreator rotativo com sistema de agitação em escala piloto é uma alternativa viável para o tratamento de solo contaminado com biodiesel bruto; b) a correção semanal da umidade mostrou-se insuficiente sendo necessário aumentar a freqüência de medição e correção deste parâmetro; c) houve um crescimento satisfatório dos microrganismos no solo, sendo que estes se mostraram capazes de degradar o contaminante; d) a remoção do biodiesel no reator proposto pode ser melhorada pelo maior controle de umidade e temperatura.

\section{AGRADECIMENTOS}

Os autores agradecem a Universidade Federal de Uberlândia e a Faculdade de Engenharia Química pela oportunidade em realizar este trabalho. Agradecem também ao apoio financeiro da CAPES, da FAPEMIG e do CNPq.

\section{REFERÊNCIAS BIBLIOGRÁFICAS}

ABNT - Associação Brasileira de Normas Técnicas. NBR 7180/81: Solo - Análise granulométrica. Rio de Janeiro: ABNT, 1984. 13p.

APHA - American Public Health Association. Standard Methods for examination of water and wastewater. Maryland, USA, $18^{\mathrm{a}}$ ed., 1992.

BENTO, F.M.; CAMARGO, F.A.O.; OKEKE, B.C.; FRANKENBERGER, W.T. Comparative bioremediation of soils contaminated with diesel oil by natural attenuation, biostimulation and bioaugmentation. Bioresource Technology, v.96, p.1049-1055, 2005.

EL FANTROUSSI, S.; AGATHOS, S.N. Is bioaugmentation a feasible strategy for pollutant removal and site remediation?. Current Opinion in Microbiology, v. 8, p. 268-275, 2005.

JACQUES, R.J.S.; SILVA K.J.; BENTO M.; CAMARGO F.A.O. Biorremediação de um solo contaminado com antraceno sob diferentes condições físicas e químicas. Ciência Rural, v. 40, p. $310-317,2010$.

LEMOS, A.L. Tratamento Biológico de Solo Contaminado por Biodiesel Bruto. 2013. $107 f$. Dissertação (Mestrado em Engenharia Química) - Faculdade de Engenharia Química, Universidade Federal de Uberlândia, Minas Gerais, 2013.

MEYER, D. D. Avaliação da Biodegradabilidade de Misturas de Diesel e de Biodiesel (BO, B20 e B100) em Dois Solos com Diferentes Granulometrias. 2011. 53f. Dissertação (Mestrado em Microbiologia Agrícola e Meio Ambiente) - Instituto de Ciências Básicas da Saúde, Universidade Federal do Rio Grande do Sul, 2011.

MOREIRA, F.M.S.; SIQUEIRA, J.O. 2006. Microbiologia e Bioquímica do Solo. 2a ed. Editora UFLA. 729p.

PREVEDELLO, C.L. Física do solo. Curitiba: Ed. Salesward-Discovery, 446 p., 1996. 
RIVALDI, J.D.; SARROUH, B.F.; FIORILO, R.; SILVA, S.S. Glicerol de biodiesel. Revista Eletrônica: Biotecnologia Ciência \& Desenvolvimento, v. 37, p. 44-51, 2007.

RIZZO, A.C.L; LEITE, S.G.F; SORIANO, A.U.; SANTOS, R.L.C; SOBRAL, L.G.S. Biorremediação de solos contaminados por petróleo: ênfase no uso de biorreatores. Rio de Janeiro: CETEM/MCT, 2007. 76p. (Série Tecnologia Ambiental, 37).

U. S. ENVIRONMENTAL PROTECTION AGENCY - (USEPA). How to Evaluate Alternative Cleanup Technologies for Underground Storage Tank Sites: A Guide for Corrective Action Plan Reviewers. Chapter XII - Enhanced Aerobic Bioremediation, EPA 510-B-94-003; EPA 510-B-95-007 and EPA 510-R-04-002, 2004, 73p.

VIEIRA, P.A. Avaliação da Biodegradação de Efluente Contendo Óleo Diesel e Gasolina Empregando Culturas Mistas. 2004. 101f. Dissertação (Mestrado em Engenharia Química) - Faculdade de Engenharia Química, Universidade Federal de Uberlândia, Minas Gerais, 2004.

VIEIRA, P.A.; VIEIRA, R.B.; DE FRANÇA, F.P.; CARDOSO, V.L. Biodegradation of effluent contaminated with diesel fuel and gasoline. Journal of Hazardous Materials, v. 140, p. 5259, 2007.

WATWOOD, M. E.; WHITE, C. S.; DAHN, C. N. Methodological modifications for accurate and efficient determination of contaminant biodegradation in unsaturated calcareous soils. Applied and Environmental Microbiology, v. 57, p.717-720, 1991. 